Published in final edited form as:

J Diabetes Complications. 2015 April ; 29(3): 343-349. doi:10.1016/j.jdiacomp.2014.12.014.

\title{
Dietary patterns associated with HbA1c and LDL cholesterol among individuals with type 1 diabetes in China
}

\section{Lindsay M. Jaacks ${ }^{a}$, Jamie Crandell ${ }^{b}$, Michelle A. Mendez ${ }^{c}$, Archana P. Lamichhane ${ }^{d}$, Wei Liu $^{\mathrm{e}}$, Linong Ji ${ }^{f}$, Shufa Dug , Wayne Rosamond ${ }^{\mathrm{h}}$, Barry M. Popkin', and Elizabeth J. Mayer- Davis}

Jamie Crandell: jbigelow@email.unc.edu; Michelle A. Mendez: mmendez@email.unc.edu; Archana P. Lamichhane: lamichha@email.unc.edu; Wei Liu: liuwei850217@163.com; Linong Ji: jiln@bjmu.edu.cn; Shufa Du:

dushufa@email.unc.edu; Wayne Rosamond: wayne_rosamond@unc.edu; Barry M. Popkin: popkin@unc.edu; Elizabeth

J. Mayer-Davis: ejmayer_davis@unc.edu

aDepartment of Nutrition, The University of North Carolina, Chapel Hill, North Carolina, United

States

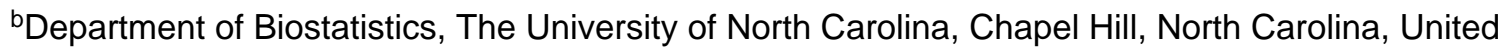
States

'Department of Nutrition, The University of North Carolina, Chapel Hill, North Carolina, United States

${ }^{\mathrm{d} D e p a r t m e n t}$ of Nutrition, The University of North Carolina, Chapel Hill, North Carolina, United States

eDepartment of Endocrinology and Metabolism, Peking University People's Hospital, Beijing, China

fDepartment of Endocrinology and Metabolism, Peking University People's Hospital, Beijing, China

9Department of Nutrition, The University of North Carolina, Chapel Hill, North Carolina, United States

hDepartment of Epidemiology, The University of North Carolina, Chapel Hill, North Carolina, United States

iDepartment of Nutrition, The University of North Carolina, Chapel Hill, North Carolina, United States

iDepartment of Nutrition, The University of North Carolina, Chapel Hill, North Carolina, United States

\footnotetext{
() 2014 Elsevier Inc. All rights reserved.

Correspondence Lindsay M. Jaacks, 135 Dauer Drive, Campus Box 7461, Chapel Hill, NC 27599, Telephone: +001 (919) 966-7218, Fax: +001 (919) 843-2011, jaacks@email.unc.edu.

Conflicts of Interest

The authors declare that they have no conflicts of interest.

Publisher's Disclaimer: This is a PDF file of an unedited manuscript that has been accepted for publication. As a service to our customers we are providing this early version of the manuscript. The manuscript will undergo copyediting, typesetting, and review of the resulting proof before it is published in its final citable form. Please note that during the production process errors may be discovered which could affect the content, and all legal disclaimers that apply to the journal pertain.
} 


\section{Abstract}

Aims-To identify dietary patterns that influence cardiometabolic risk among individuals with type 1 diabetes (T1D) in China.

Methods-Data are from a cross-sectional study of T1D in China (n=99). Dietary intake was assessed using three 24-hour recalls. Reduced rank regression was used to identify dietary patterns from a set of 20 food groups that maximized the explained variation in glycated hemoglobin A1c (HbA1c) and low-density lipoprotein (LDL) cholesterol.

Results-Dietary pattern 1 was characterized by low intakes of wheat products and high-fat cakes, and high intakes of beans and pickled vegetables. Dietary pattern 2 was characterized by low intakes of high-fat cakes, nuts/seeds, fish/shellfish, and teas/coffee, and high intakes of rice and eggs. Participants in the highest tertile of dietary pattern 1 had significantly $(\mathrm{p}<0.05)$ higher HbA1c and LDL cholesterol compared to participants in the lowest tertile: mean difference in $\mathrm{HbA} 1 \mathrm{c}$ was 1.0 percentage point $(11 \mathrm{mmol} / \mathrm{mol})$ and in $\mathrm{LDL}$ cholesterol was $0.36 \mathrm{mmol} / \mathrm{L}$ after adjustment for age and household income. Dietary pattern 2 was not associated with HbA1c or LDL cholesterol.

Conclusions-We identified a dietary pattern that is significantly related to HbA1c and LDL cholesterol. These findings provide support for behavioral strategies to prevent complications in individuals with T1D in China.

\section{Keywords}

reduced rank regression; dietary patterns; glycemic control; type 1 diabetes; China

\section{Introduction}

Improvements in treatment over the past 30 years have reduced the risk of cardiovascular disease (CVD) and all-cause mortality among individuals with type 1 diabetes (T1D) [1-3]; however, CVD and mortality rates remain significantly higher among adults with T1D relative to the general population $[2,4]$. Furthermore, substantial geographic variation in mortality rates even among developed countries $[5,6]$ suggests that improvements in T1D treatment have not been shared equally across the globe.

Since the completion of the landmark Diabetes Control and Complications Trial [7], treatment efforts to reduce CVD and other complications among individuals with T1D have focused on intensifying self-monitoring of blood glucose (SMBG) and insulin dose adjustments to achieve tight glycemic control (HbA1c < $7 \%$ [53 mmol $/ \mathrm{mol}]$, which corresponds to a 3-month average blood glucose of $8.6 \mathrm{mmol} / \mathrm{L}$ ) [8,9]. However, these intensive treatment regimens are uncommon in many parts of the world including China where the median number of times per day patients monitor is 0.4 [10], in stark contrast to the recommended 6 to 8 [8]. China has the largest number of individuals with T1D in the Western Pacific Region [11] and there is some evidence to suggest that the burden of comorbidities in this population is higher than that in the United States: 2001-2002 data from two centers in China on hypertension in youth $<15$ years old with T1D estimated a hypertension prevalence of $24 \%$ [12] compared to 5.9\% among youth 3 to 17 years old with 
T1D in the United States [13]. Strategies to reduce CVD and other complications in this high-risk population are urgently needed.

Dietary factors including, for example, high fiber intake [14], are prospectively associated with reduced risk of CVD and all-cause mortality among European adults with T1D. Dietary intake may therefore represent an important point of intervention to improve health outcomes for this population. Reduced rank regression (RRR) has emerged over the past 10 years as a method to identify disease-specific dietary patterns [15]. The objective of this analysis was to use RRR to identify dietary patterns maximizing the explained variation in two key cardiometabolic risk factors, glycated hemoglobin A1c (HbA1c) and low-density lipoprotein (LDL) cholesterol, among individuals with T1D in China. Results could be used to inform lifestyle interventions to prevent or delay long-term complications of T1D in China, thus reducing healthcare costs and improving patient outcomes.

\section{Subjects, Materials, and Methods Sample population}

The 3C Study was an epidemiological study of the coverage, cost, and care of T1D in China [16]. The 3C Nutrition Ancillary Study (3CNAS), was conducted, on average, (mean \pm SD) $1.6 \pm 0.2$ years later and expanded the 3C Study to include detailed, cross-sectional information on dietary intake. 3C Study participants who met the following criteria were eligible for 3CNAS: Beijing resident, $\geq 12$ years old, no severe diabetes complications (i.e. advanced micro- and macrovascular complications, including nephropathy and stroke), and in-service telephone number available. A total of 195 3C Study participants met these criteria. Of these, 72 (37\%) refused to participate, 23 (12\%) dropped out before the 3CNAS visit, and $1(0.5 \%)$ refused to complete the fasting blood draw. The final sample size for this analysis was therefore $n=99$. There were no differences in diabetes duration, sex, education, or urban versus rural residence between those who participated and those who refused or dropped out (all $\mathrm{p}>0.05)$. However, those who participated tended to be older $(\mathrm{p}=0.001)$, have higher household incomes ( $\mathrm{p}=0.05)$, and be retired or unemployed $(\mathrm{p}=0.06)$ and married or cohabitating $(\mathrm{p}=0.02)$.

All procedures were approved by the University of North Carolina Office of Human Research Ethics and the Peking University Biomedical Institutional Review Board, and all participants provided written informed consent ( $\geq 18$ years old) or written parent permission and participant assent $(<18$ years old).

\section{Data collection}

Self-reported demographic, socioeconomic, and physical activity data were collected during the 3C Study visit. Physical activity was reported as the number of days in the past week that the individual participated in at least 30 minutes of physical activity, and was categorized as 0-3 days/week, 4-6 days/week, and 7 days/week. Diabetes duration was calculated as the period from July 1 of the year of diagnosis (because the 3C Study only queried year of diagnosis) to the 3 CNAS visit. 
A fasting blood sample was collected by venipuncture during the 3CNAS visit and biomarkers (HbA1c, high-density lipoprotein (HDL) cholesterol, LDL cholesterol, total cholesterol, and triglycerides) were assessed using standardized laboratory procedures. Specifically, HbA1c was measured in whole blood with an automated high-performance liquid chromatography system (Primus Ultra ${ }^{2}$, Trinity Biotech, Bray, Co Wicklow, Ireland). Blood samples for lipid analysis, including LDL cholesterol, were immediately centrifuged at 2000 revolutions per minute (RPM) for 15 minutes and the serum was stored at $-80^{\circ} \mathrm{C}$ for batch analysis using an automated Hitachi-008 system (Hitachi, Chiyoda, Tokyo, Japan).

Also during the 3CNAS visit, participants were asked if they had ever been taught carbohydrate counting and if yes, how often they use it. Information on insulin administration method, type, and dose, and SMBG were also collected during this visit. Four insulin regimens were defined as follows: (1) multiple daily injections (MDI; $\geq 3$ injections) that included glargine or detemir ("MDI: With basal"), (2) continuous subcutaneous infusion ("Pump"), (3) MDI with any insulin types excluding glargine and detemir ("MDI: No basal"), and (4) 1-2 injections per day of any insulin types ("1-2 injections").

At the end of the 3CNAS visit, participants were trained by dietitians to record their dietary intake on food records provided in an introductory packet. Emphasis was placed on estimating portion sizes using food samples, an electronic scale, and a culturally appropriate portion size picture guide. On average, beginning (mean $\pm \mathrm{SD}$ ) $3.0 \pm 2.8$ days after this visit, three (two weekday and one weekend day) telephone administered 24-hour dietary recalls were completed with the assistance of food records. Telephone administration of the recalls was previously validated against in-person administration in a subset of participants [17]. The average time between recalls one and two was (mean $\pm \mathrm{SD}$ ) $2.5 \pm 1.9$ days and between recalls two and three was (mean \pm SD) $2.6 \pm 2.0$ days. Two participants did not complete a third recall; their dietary intake was calculated as the average of the two available days. One participant completed two weekend day recalls and one weekday recall; his intanke was calculated as the average of all three available days. All recalls were administered using a two-pass approach: (1) collect a detailed food list and (2) review and confirm the detailed food list. During the first pass, an outline of the previous day's intake was collected with guidance from the completed food record. Interviewers probed for meal type, preparation setting, preparation method and detailed information on any additions to the food, food type, brand names, and portion size. During the second pass, the detailed food list was re-read and missing foods, beverages, and eating occasions were probed.

The 24-hour recall food lists were converted into 42 nutritionally meaningful food groups by a collaborative working group that included researchers at The University of North Carolina, Chapel Hill, and the Chinese Institute of Nutrition and Food Safety [18]. Four food groups (plant oils, salt, other spices \& condiments, and herbs \& other functional foods) were excluded because of the measurement error associated with estimating added oil, salt, and spices, which were not the focus of this analysis. Consistent with previous RRR analyses of non-diabetic Chinese adults [19], alcohol was excluded because it was only consumed by males. Food groups with $<10 \%$ consumers (Supplemental Table 1) were also excluded because of their lack of variability in this sample population. Where appropriate given behavioral and cultural considerations, food groups were combined. These combinations 
included starchy tubers and starchy tuber products; beans and bean products; low- and highbeta carotene vegetables; fresh or canned fruit and dried fruit; milk and milk products; lowand high-fat meat and meat products; low- and high-fat poultry; and fish and shellfish. A total of 20 food groups were considered for the RRR analysis (Supplemental Table 2). A summary of the food group inclusion process is provided in Supplemental Figure 1.

Most food groups had a large proportion of non-consumers and therefore all food groups were dichotomized for the RRR analysis. Food groups with $\geq 80 \%$ consumers were dichotomized as below versus above the median, and food groups with $\geq 10 \%$ but $<80 \%$ consumers were dichotomized as non-consumers versus consumers.

\section{Statistical analysis}

We used RRR to identify dietary patterns maximizing the explained variation in cardiometabolic risk factors [15]. Twenty food groups adjusted for total energy intake using the density method [20] were used as predictors. Total energy intake was calculated using the Chinese Food Composition Tables [21,22]. Two predefined cardiometabolic risk factors, $\mathrm{HbA1c}$ and LDL cholesterol, log-transformed to improve normality, were used as responses. $\mathrm{HbA1c}$ and LDL cholesterol were chosen because they are well established risk factors for CVD and diabetes-related complications in individuals with T1D [23-30]. RRR produces as many factors ("dietary patterns") as there are responses, and consequently two factors were obtained. To characterize the factors, food groups with factor loadings $\searrow 0.25$ were considered. Using PLS (partial least squares) procedure in SAS software specifying method=RRR, linear functions of the 20 predictors (known as "dietary pattern scores") were derived which maximized the proportion of explained variation in the set of two responses. These dietary pattern scores were then categorized into tertiles. The explained proportion of score variation was calculated for each food group strongly associated (factor loadings $\searrow$.25) with the dietary patterns as the product of the standardized score parameter, the correlation coefficient with the dietary pattern score, and $100 \%$. Results are presented in order of decreasing explained proportion of score variation.

In order to improve RRR model fit, a backwards elimination approach was used to reduce the number of predictor variables. The food group that was explained the least by the dietary patterns was eliminated first and the RRR was re-fitted. This procedure was repeated until all food groups with $<5 \%$ of their variance explained by the dietary patterns, and consequently those that did not load heavily on either factor, were eliminated. Results were largely consistent compared to the full RRR model (20 predictors) with respect to food group loadings and associations with the response variables at this point, and therefore only the nine remaining predictors (rice, wheat products, high-fat cakes, beans $\&$ bean products, nuts $\&$ seeds, pickled vegetables, eggs, fish $\&$ shellfish, and teas $\&$ coffee) were included in the final RRR.

Differences in demographic and clinical characteristics across tertiles of the dietary pattern scores were assessed using chi-square tests for categorical variables and Kruskal-Wallis tests for continuous variables. Furthermore, because socio-demographic characteristics may partially explain observed associations between the RRR-derived dietary patterns and the responses, we calculated mean values of the cardiometabolic risk factors across the dietary 
pattern score tertiles with adjustment for age and household income using analysis of covariance (ANCOVA). Potential confounding variables that met the following criteria were included in the final models: (1) associated with the predictor variables (food groups) at $\mathrm{p}$ $<0.10$, (2) associated with the response variables (HbA1c and LDL cholesterol) at $\mathrm{p}<0.10$, and (3) not affected by the exposure [31]. Age and household income met these criteria (data not shown) and were therefore included in all multivariable analyses.

After identifying dietary patterns associated with $\mathrm{HbA1c}$ and LDL cholesterol, we explored bivariate associations between the food groups identified as being important (factor loadings $\searrow$.25) in the RRR and each of these risk factors.

Values presented are median $\left(25^{\text {th }}\right.$ percentile, $75^{\text {th }}$ percentile $)$ or $\mathrm{n}(\%)$. All statistical analyses were conducted in SAS 9.2 (SAS Institute, Cary, North Carolina).

\section{Results}

Participants $\left(n=99 ; 55 \%\right.$ male) were (median $\left[25^{\text {th }}\right.$ percentile, $75^{\text {th }}$ percentile $\left.]\right) 43.6$ ( 28.4 , $55.0)$ years old and had a diabetes duration of $7.8(4.8,17.8)$ years. The HbAlc for the sample was $7.9 \%(7.0,9.0)(62.8 \mathrm{mmol} / \mathrm{mol}[53.0,74.9])$, and the LDL cholesterol for the sample was $2.3 \mathrm{mmol} / \mathrm{L}(1.9,2.8)$.

Dietary pattern 1 was characterized by low intakes of wheat products and high-fat cakes, and high intakes of beans \& bean products and pickled vegetables (Table 1). These four food groups together explained $83 \%$ of the variation in the first dietary pattern score with the most important contributors being high-fat cakes (36\%) and wheat products (33\%). Dietary pattern 2 was characterized by low intakes of high-fat cakes, nuts $\&$ seeds, fish $\&$ shellfish, and teas \& coffee, and high intakes of rice and eggs. These six food groups together explained $90 \%$ of the variation in the second dietary pattern score with the most important contributors being teas \& coffee (33\%) and fish \& shellfish (21\%).

The first RRR-identified dietary pattern explained 5.9\% of the variation in HbA1c, 9.8\% in LDL cholesterol, and $7.9 \%$ of the total variation in both cardiometabolic risk factors. The second dietary pattern explained $7.1 \%$ of the variation in HbA1c, $4.2 \%$ in LDL cholesterol, and $5.7 \%$ of the total variation in both cardiometabolic risk factors. HbA1c and LDL cholesterol were not significantly correlated in this sample (Pearson Correlation Coefficient $=0.01, \mathrm{p}=0.89$ ).

There were few significant differences across tertiles of the dietary pattern scores in terms of socio-demographic and diabetes self-management characteristics (Table 2). Participants in the highest tertile of dietary pattern 1 score had marginally significantly longer diabetes durations $(\mathrm{p}=0.09)$. The significant association observed with insulin regimen was equivocal: participants in the lowest and highest tertile of dietary pattern 1 score were more likely to be on "MDI: With basal" or "pumps," and less likely to be on "MDI: No basal" or "1-2 injections" compared to participants in the middle tertile.

Participants in the highest tertile of dietary pattern 1 score had significantly higher $\mathrm{HbA} 1 \mathrm{c}$ (Fig. 1A) and LDL cholesterol (Fig. 2A) levels compared to participants in the lowest tertile 
after adjustment for age and household income: mean difference in $\mathrm{HbA1c}$ was 1.0 percentage point $(11 \mathrm{mmol} / \mathrm{mol})$ and in $\mathrm{LDL}$ cholesterol was $0.36 \mathrm{mmol} / \mathrm{L}$. There were no significant differences in HbA1c or LDL cholesterol in either unadjusted or adjusted analyses across tertiles of dietary pattern 2 score (Fig. 1B and Fig. 2B). Results were consistent with additional adjustment for physical activity and carbohydrate counting (data not shown).

In bivariate analyses estimating the association between key food groups identified in the RRR analysis and the risk factors (Table 3), only high-fat cakes were associated with HbA1c: consumers had significantly lower HbA1c compared to non-consumers ( $\mathrm{p}=0.008$ ). Consumers of wheat products had significantly lower LDL cholesterol compared to nonconsumers $(\mathrm{p}=0.03)$, and consumers of fish $\&$ shellfish and teas $\&$ coffee had marginally significantly higher LDL cholesterol compared to non-consumers ( $\mathrm{p}=0.08$ and $\mathrm{p}=0.05$, respectively).

\section{Discussion}

We identified a dietary pattern characterized by low intakes of wheat products and high-fat cakes, and high intakes of beans \& bean products and pickled vegetables that was significantly positively associated with HbA1c and LDL cholesterol in a cohort of adolescents and adults with T1D in China: the mean adjusted difference between the highest and lowest tertile of the dietary pattern score for HbA1c was one percentage point and for LDL cholesterol it was $0.36 \mathrm{mmol} / \mathrm{L}$. In a meta-analysis of prospective cohort studies, a onepercentage point increase in HbA1c was associated with an increase in CVD risk of $18 \%$ [32], thus this finding is clinically meaningful. Furthermore, a difference in LDL cholesterol of $0.8 \mathrm{mmol} / \mathrm{L}$ can move an individual from near optimal to borderline high risk, and therefore our observed difference in LDL cholesterol of approximately $0.4 \mathrm{mmol} / \mathrm{L}$ was also clinically important. These observations improve our understanding of dietary factors influencing CVD risk among individuals with T1D living in a country undergoing rapid changes in nutrition and health $[33,34]$.

Participants in the highest tertile of the dietary pattern 1 score were more likely to be consumers of pickled vegetables, which tend to be high in salt, and these participants also had higher LDL cholesterol levels. This is consistent with a study finding an association between self-reported consumption of salted food and hyperlipidemia among Chinese men [35]. However, results from the FinnDiane Study suggest that the relationship between dietary sodium and mortality among adults with T1D may be U-shaped with both low and high intakes associated with increased mortality [36]. Furthermore, an inverse association was observed between sodium excretion and end-stage renal disease in this cohort, supporting a cautionary approach to salt restriction [36]. Given these mixed results, clinical trials are warranted to explore the effects of dietary sodium on cardiometabolic outcomes in this population.

Interestingly, a low intake of high-fat cakes was an important aspect of dietary pattern 1, which was ultimately associated with poor risk factor status. Indeed, "high-fat cakes" was the only food group significantly associated with $\mathrm{HbA1c}$ in bivariate analyses. This 
observation makes sense given that nearly all T1D patients in China report having been told to limit their sweet intake (for example, desserts, non-diet sodas, and candy), and "sugar free" products are increasingly available at food retailers in China. Individuals that adhere to recommendations may choose sugarless cakes that are high in fat over low-fat cakes that are high in sugar. While not statistically significant, a greater proportion of low-fat cake consumers were in the highest tertile of dietary pattern 1 (and therefore had lower high-fat cake intakes): $45 \%$ compared to $26 \%$ in the lowest tertile of dietary pattern 1 . Together, these observations may explain the inverse association between high-fat cake consumption and $\mathrm{HbA} 1 \mathrm{c}$ in this analysis.

In contrast to a small $(n=12)$ crossover dietary intervention trial of a soy diet in young adults with T1D and hyperfiltration (defined as glomular filtration rates $>120 \mathrm{~mL} / \mathrm{min} / 1.73 \mathrm{~m}^{2}$ ) [37], and a meta-analysis of intervention trials in adults without T1D [38], which both found a soy-based diet high in isoflavones reduced LDL cholesterol, we found that a dietary pattern characterized by high bean $\&$ bean product intakes (typically soy-based products such as tofu) was associated with higher LDL cholesterol and HbA1c. This result was unexpected, though it is important to consider that only $5.8 \%$ of the variability in the dietary pattern score was explained by this food group. More research is needed to understand the effects of bean $\&$ bean product intake on health in this population.

To our knowledge, only one study has derived dietary patterns using RRR in a population of individuals with T1D [39]. Using data from the SEARCH for Diabetes in Youth Study, a dietary pattern characterized by high intakes of eggs, sweetened coffee and tea, sweetened soda/fruit-flavored drinks, diet soda, potatoes, and high-fat meat, and low intakes of sweets/ desserts and low-fat dairy was positively associated with markers of CVD risk (LDL cholesterol, triglyceride, systolic blood pressure, HbA1c, C-reactive protein, and waist circumference) [39]. The differences observed between this dietary pattern and the dietary pattern derived in our analysis are likely the result of a combination of factors including different sample populations (different continent of residence, age, and disease duration), different response variables (disease biomarkers), and different predictor variables (due to cultural differences in dietary intake). Nonetheless, the similarities are worth mentioning here, particularly high intakes of eggs, which was an important food group in both studies. Evidence relating egg consumption to CVD risk is equivocal: while a recent meta-analysis found that egg consumption is related to increased risk of coronary heart disease among adults with type 2 diabetes (relative risk [95\% confidence interval] comparing highest and lowest egg intake 1.54 [1.14, 2.09]), there was no association among non-diabetic adults [40]. More research on dietary patterns in diverse populations is needed to improve our understanding of the effects of foods and beverages on disease outcomes.

The objective of RRR is not to describe dietary patterns that reflect either dietary recommendations or cultural patterns described in the literature, but rather to identify dietary patterns important in the development of disease. Nonetheless, an important challenge of interpreting this analysis is that the dietary patterns identified by RRR do not necessarily represent patterns of foods and beverages actually eaten together in the sample population. Furthermore, this study was cross-sectional and we cannot rule out reverse causality: the process of recording and recalling dietary intake could differentially affect reporting 
between those at high-risk and those at low-risk of CVD. However, purposeful dietary misreporting may be uncommon in China [41]. In our study, these biases were addressed by using interviewers who were not the participants' health care providers and training them to use a standardized protocol and neutral probes [42], as well as emphasizing in conversations with participants that we were interested in understanding what individuals with T1D usually eat and that it was important that they not change their dietary habits. Finally in regard to the dietary assessment method, we used telephone interviews to conduct the 24hour dietary recalls, and therefore were not able to precisely measure salt, spice, and condiment intake within the household.

Another limitation of this study is that more detailed data on circulating lipids, such as apolipoprotein B, small-dense LDL particles, oxidized LDL, and advanced glycation end products-modified LDL, as well as current blood pressure, were not available. Given that these risk factors have also been implicated in the development of vascular disease in individuals with T1D [43], future research should explore their association with dietary intake in more diverse populations. Finally, we had a relatively small sample size, which limits the generalizability of our findings. However, this is the largest sample of individuals with T1D in a developing country for which comprehensive dietary data are available, and therefore these results provide invaluable insight into potential areas for future research.

In summary, a dietary pattern was identified that is strongly related to two established risk factors for serious, chronic complications of T1D. These findings provide support for development of behavioral strategies to prevent complications including CVD in adolescents and adults with T1D in China.

\section{Supplementary Material}

Refer to Web version on PubMed Central for supplementary material.

\section{Acknowledgments}

3CNAS is indebted to the individuals whose participation made this study possible, and to our interviewers: Yang Xiao, Jing Lv, Wenjia Yang, Jia Liu, Han Feifei, and Lihua Zhang. We would like to acknowledge Helen McGuire, Principle Investigator of the Care and Education arm of the 3C Study: Coverage, Cost, and Care of Type 1 Diabetes in China. The 3C Study was a collaborative effort of the International Diabetes Federation and the Chinese Diabetes Society.

3CNAS was supported by funding from the Sanofi Global Scholars Program, the Fogarty International Center of the National Institutes of Health (5D43TW009077), and the National Center for Advancing Translational Sciences (ULTR000083). None of the aforementioned funding sources had a role in the design, analysis, or writing of this article.

\section{References}

1. Harjutsalo V, Forsblom C, Groop PH. Time trends in mortality in patients with type 1 diabetes: nationwide population based cohort study. BMJ. 2011; 343:d5364. [PubMed: 21903695]

2. Livingstone SJ, Looker HC, Hothersall EJ, Wild SH, Lindsay RS, Chalmers J, et al. Risk of cardiovascular disease and total mortality in adults with type 1 diabetes: Scottish Registry Linkage Study. PLoS Med. 2012; 9:e1001321. [PubMed: 23055834] 
3. Nishimura R, LaPorte RE, Dorman JS, Tajima N, Becker D, Orchard TJ. Mortality trends in type 1 diabetes. The Allegheny County (Pennsylvania) Registry 1965-1999. Diabetes Care. 2001; 24:823827. [PubMed: 11347737]

4. Soedamah-Muthu SS, Fuller JH, Mulnier HE, Raleigh VS, Lawrenson RA, Colhoun HM. All-cause mortality rates in patients with type 1 diabetes mellitus compared with a non-diabetic population from the UK general practice research database, 1992-1999. Diabetologia. 2006; 49:660-666. [PubMed: 16432708]

5. Matsushima M, LaPorte RE, Maruyama M, Shimizu K, Nishimura R, Tajima N. Geographic variation in mortality among individuals with youth-onset diabetes mellitus across the world. Diabetologia. 1997; 40:212-216. [PubMed: 9049483]

6. Morrish NJ, Wang SL, Stevens LK, Fuller JH, Keen H. Mortality and causes of death in the WHO Multinational Study of Vascular Disease in Diabetes. Diabetologia. 2001; 44:S14-21. [PubMed: 11587045]

7. Diabetes Control and Complications Trial Research Group. The Effect of intensive treatment of diabetes on the development and progression of long-term complications in insulin-dependent diabetes mellitus. N Engl J Med. 1993; 329:977-986. [PubMed: 8366922]

8. American Diabetes Association. Standards of medical care in diabetes-2014. Diabetes Care. 2014; 37:S14-S80. [PubMed: 24357209]

9. Nathan DM, Kuenen J, Borg R, Zheng H, Schoenfeld D, Heine RJ, et al. Translating the A1C assay into estimated average glucose values. Diabetes Care. 2008; 31:1473-1478. [PubMed: 18540046]

10. Li J, Yang D, Yan J, Huang B, Zhang Y, Weng J. Secondary Diabetic ketoacidosis and severe hypoglycaemia in patients with established type 1 diabetes mellitus in China: a multicentre registration study. Diabetes Metab Res Rev. 2014 Epub ahead of print.

11. International Diabetes Federation. Diabetes Atlas. 5. Brussels: International Diabetes Federation; 2011.

12. Craig ME, Jones TW, Silink M, Ping YJ. Diabetes care, glycemic control, and complications in children with type 1 diabetes from Asia and the Western Pacific Region. J Diabetes Complications. 2007; 21:280-287. [PubMed: 17825751]

13. Rodriguez BL, Dabelea D, Liese AD, Fujimoto W, Waitzfelder B, Liu L, et al. Prevalence and correlates of elevated blood pressure in youth with diabetes mellitus: the Search for Diabetes in Youth Study. J Pediatr. 2010; 157:245-251.e1. [PubMed: 20394942]

14. Schoenaker DA, Toeller M, Chaturvedi N, Fuller JH, Soedamah-Muthu SS. EURODIAB Prospective Complications Study Group. Dietary saturated fat and fibre and risk of cardiovascular disease and all-cause mortality among type 1 diabetic patients: the EURODIAB Prospective Complications Study. Diabetologia. 2012; 55:2132-2141. [PubMed: 22526612]

15. Hoffmann K, Schulze MB, Schienkiewitz A, Nöthlings U, Boeing H. Application of a new statistical method to derive dietary patterns in nutritional epidemiology. Am J Epidemiol. 2004; 159:935-944. [PubMed: 15128605]

16. McGuire H, Kissimova-Skarbek K, Whiting D, Ji L. The 3C study: coverage cost and care of type 1 diabetes in China--study design and implementation. Diabetes Res Clin Pract. 2011; 94:307310. [PubMed: 22056720]

17. Jaacks LM, Liu W, Ji L, Mendez M, Du S, Crandell J, et al. Diabetes nutrition therapy and dietary intake among individuals with type 1 diabetes in China. Diabet Med. 2014 In press.

18. Popkin BM, Lu B, Zhai F. Understanding the nutrition transition: measuring rapid dietary changes in transitional countries. Public Health Nutr. 2002; 5:947-953. [PubMed: 12633520]

19. Batis C, Mendez MA, Sotres-Alvarez D, Gordon-Larsen P, Popkin B. Dietary pattern trajectories during 15 years of follow-up and HbA1c, insulin resistance and diabetes prevalence among Chinese adults. J Epidemiol Community Health. 2014; 68:773-779. [PubMed: 24729424]

20. Willett WC, Howe GR, Kushi LH. Adjustment for total energy intake in epidemiologic studies. Am J Clin Nutr. 1997; 65:1220S-1228S. [PubMed: 9094926]

21. Institute of Nutrition and Food Hygiene, ed. Chinese Food Composition Tables, Book 1. 2. Beijing: People's Medical Publishing House; 2009.

22. Institute of Nutrition and Food Hygiene, ed. Chinese Food Composition Tables, Book 2. 1. Beijing: People's Medical Publishing House; 2004. 
23. Schoenaker DA, Simon D, Chaturvedi N, Fuller JH, Soedamah-Muthu SS. EURODIAB Prospective Complications Study Group. Glycemic control and all-cause mortality risk in type 1 diabetes patients: the EURODIAB Prospective Complications Study. J Clin Endocrinol Metab. 2014 jc20132824.

24. Eeg-Olofsson K, Cederholm J, Nilsson PM, Zethelius B, Svensson AM, Gudbjornsdottir S, et al. Glycemic control and cardiovascular disease in 7,454 patients with type 1 diabetes: an observational study from the Swedish National Diabetes Register (NDR). Diabetes Care. 2010; 33:1640-1646. [PubMed: 20424222]

25. Nathan DM, Cleary PA, Backlund JY, Genuth SM, Lachin JM, Orchard TJ, et al. Intensive diabetes treatment and cardiovascular disease in patients with type 1 diabetes. N Engl J Med. 2005; 353:2643-2653. [PubMed: 16371630]

26. Grundy SM, Cleeman JI, Merz CN, Brewer HB, Clark LT, Hunninghake DB, et al. Implications of recent clinical trials for the National Cholesterol Education Program Adult Treatment Panel III guidelines. Circulation. 2004; 110:227-239. [PubMed: 15249516]

27. Grauslund J, Jorgensen TM, Nybo M, Green A, Rasmussen LM, Sjolie AK. Risk factors for mortality and ischemic heart disease in patients with long-term type 1 diabetes. J Diabetes Complications. 2010; 24:223-228. [PubMed: 19577486]

28. Grauslund J. Long-term mortality and retinopathy in type 1 diabetes. Acta Ophthalmol. 2010; 88:1-14. [PubMed: 20500731]

29. Snell-Bergeon JK, Hokanson JE, Jensen L, MacKenzie T, Kinney G, Dabelea D, et al. Progression of coronary artery calcification in type 1 diabetes: the importance of glycemic control. Diabetes Care. 2003; 26:2923-2928. [PubMed: 14514603]

30. Soedamah-Muthu SS, Chaturvedi N, Witte DR, Stevens LK, Porta M, Fuller JH, et al. Relationship between risk factors and mortality in type 1 diabetic patients in Europe: the EURODIAB Prospective Complications Study (PCS). Diabetes Care. 2008; 31:1360-1366. [PubMed: 18375412]

31. Rothman, KJ.; Greenland, S.; Lash, TL., editors. Modern epidemiology. 3. Philadelphia: Lippincott Williams \& Wilkins; 2008.

32. Selvin E, Marinopoulos S, Berkenblit G, Rami T, Brancati FL, Powe NR, et al. Meta-analysis: glycosylated hemoglobin and cardiovascular disease in diabetes mellitus. Ann Intern Med. 2004; 141:421-431. [PubMed: 15381515]

33. Popkin BM, Horton S, Kim S, Mahal A, Shuigao J. Trends in diet, nutritional status, and dietrelated noncommunicable diseases in China and India: the economic costs of the nutrition transition. Nutr Rev. 2001; 59:379-390. [PubMed: 11766908]

34. Popkin BM, Keyou G, Zhai F, Guo X, Ma H, Zohoori N. The nutrition transition in China: a crosssectional analysis. Eur J Clin Nutr. 1993; 47:333-346. [PubMed: 8319669]

35. Deng B, Luo T, Huang Y, Shen T, Ma J. Prevalence and determinants of hyperlipidemia in moderate altitude areas of the Yunnan-Kweichow plateau in Southwestern China. High Alt Med Biol. 2012; 13:13-21. [PubMed: 22429228]

36. Thomas MC, Moran J, Forsblom C, Harjutsalo V, Thorn L, Ahola A, et al. The association between dietary sodium intake, ESRD, and all-cause mortality in patients with type 1 diabetes. Diabetes Care. 2011; 34:861-866. [PubMed: 21307382]

37. Stephenson TJ, Setchell KD, Kendall CW, Jenkins DJ, Anderson JW, Fanti P. Effect of soy protein-rich diet on renal function in young adults with insulin-dependent diabetes mellitus. Clin Nephrol. 2005; 64:1-11. [PubMed: 16047639]

38. Taku K, Umegaki K, Sato Y, Taki Y, Endoh K, Watanabe S. Soy isoflavones lower serum total and LDL cholesterol in humans: a meta-analysis of 11 randomized controlled trials. Am J Clin Nutr. 2007; 85:1148-1156. [PubMed: 17413118]

39. Lamichhane AP, Liese AD, Urbina EM, Crandell JL, Jaacks LM, Dabelea D, et al. Associations of dietary intake patterns identified using reduced rank regression with markers of arterial stiffness among youth with type 1 diabetes. Eur J Clin Nutr. 2014 Epub ahead of print.

40. Rong Y, Chen L, Zhu T, Song Y, Yu M, Shan Z, et al. Egg consumption and risk of coronary heart disease and stroke: dose-response meta-analysis of prospective cohort studies. BMJ. 2013; 346:e8539. [PubMed: 23295181] 
41. Yao M, McCrory MA, Ma G, Tucker KL, Gao S, Fuss P, et al. Relative influence of diet and physical activity on body composition in urban Chinese adults. Am J Clin Nutr. 2003; 77:14091416. [PubMed: 12791617]

42. Nederhof AJ. Methods of coping with social desirability bias: a review. Eur J Soc Psychol. 1985; 15:263-280.

43. Lopes-Virella MF, Hunt KJ, Baker NL, Lachin J, Nathan DM, Virella G, et al. Levels of oxidized LDL and advanced glycation end products-modified LDL in circulating immune complexes are strongly associated with increased levels of carotid intima-media thickness and its progression in type 1 diabetes. Diabetes. 2011; 60:582-589. [PubMed: 20980456] 

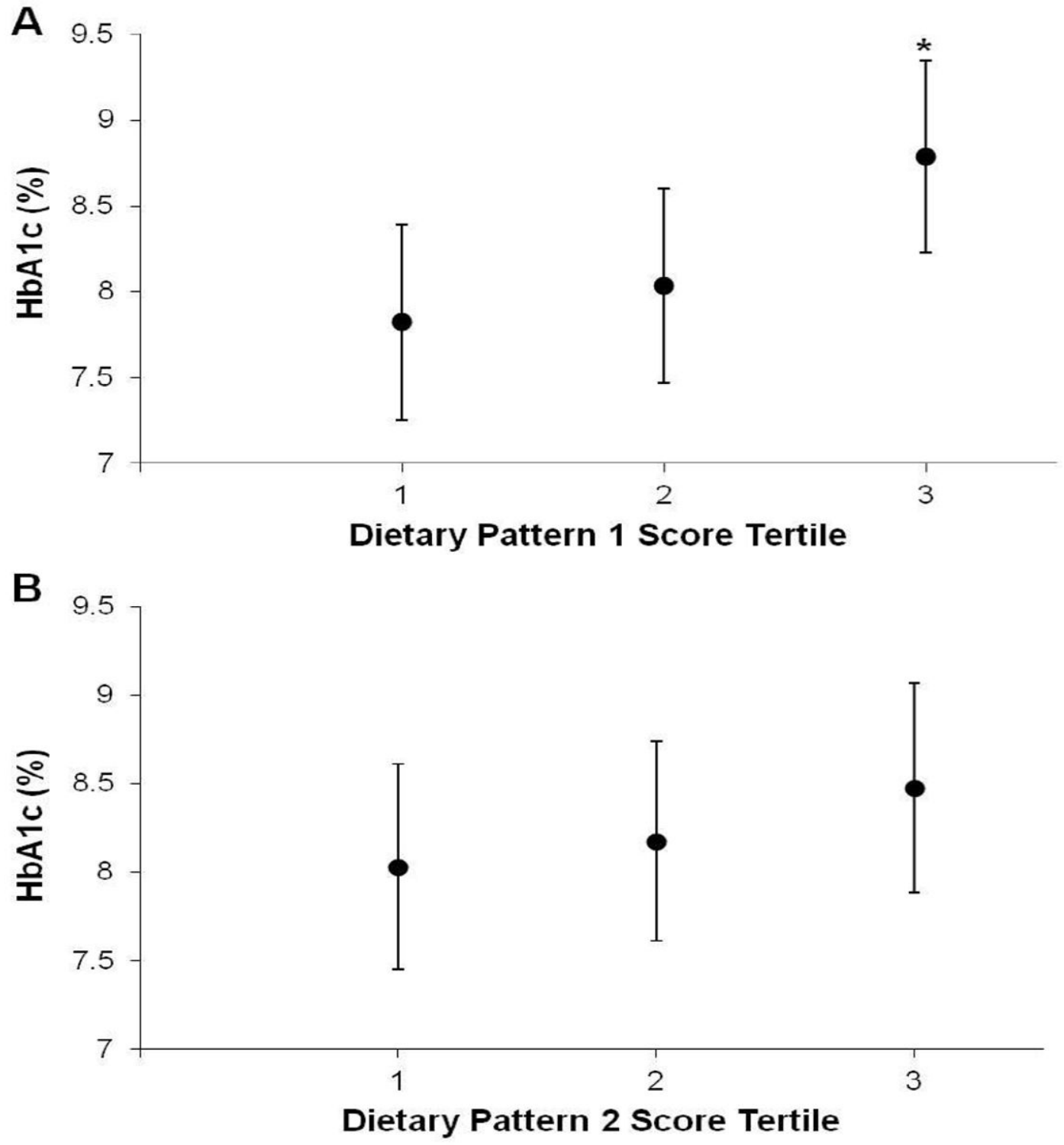

Figure 1.

Mean adjusted $\mathrm{HbA1c}(\%)$ and $95 \%$ confidence intervals from analysis of covariance (ANCOVA) according to tertiles of $\mathrm{A}$ ) reduced rank regression (RRR)-derived dietary pattern 1 score and B) RRR-derived dietary pattern 2 score among individuals with type 1 diabetes in China ( $n=99)$. Adjusted for age and household income. ${ }^{*} \mathrm{P}<0.05$ comparing $1^{\text {st }}$ and $3^{\text {rd }}$ tertiles 

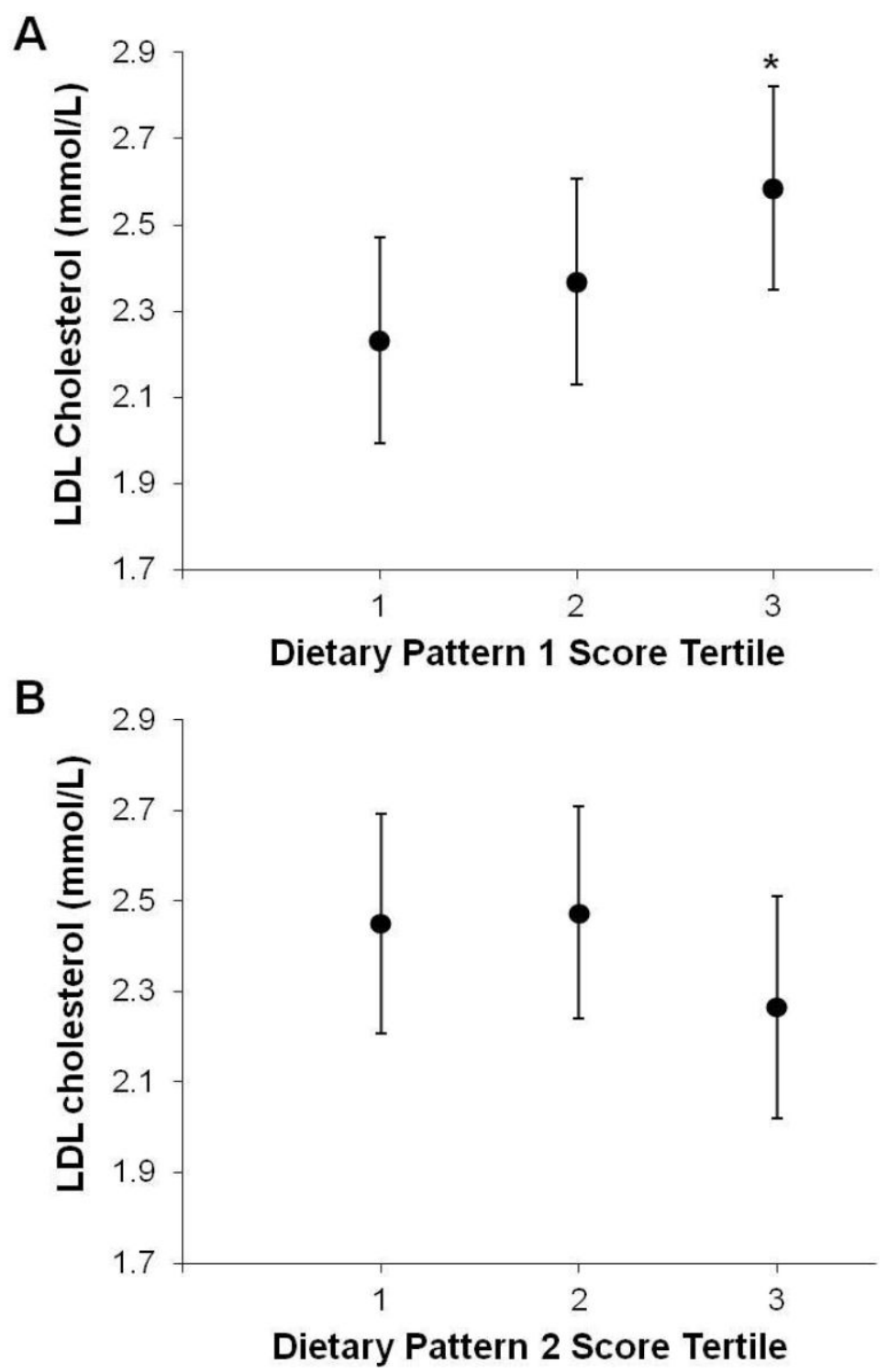

Figure 2.

Mean adjusted LDL cholesterol ( $\mathrm{mmol} / \mathrm{L})$ and $95 \%$ confidence intervals from analysis of covariance (ANCOVA) according to tertiles of A) reduced rank regression (RRR)-derived dietary pattern 1 score and B) RRR-derived dietary pattern 2 score among individuals with type 1 diabetes in China ( $n=99$ ). Adjusted for age and household income. $* \mathrm{P}<0.05$ comparing $1^{\text {st }}$ and $3^{\text {rd }}$ tertiles 


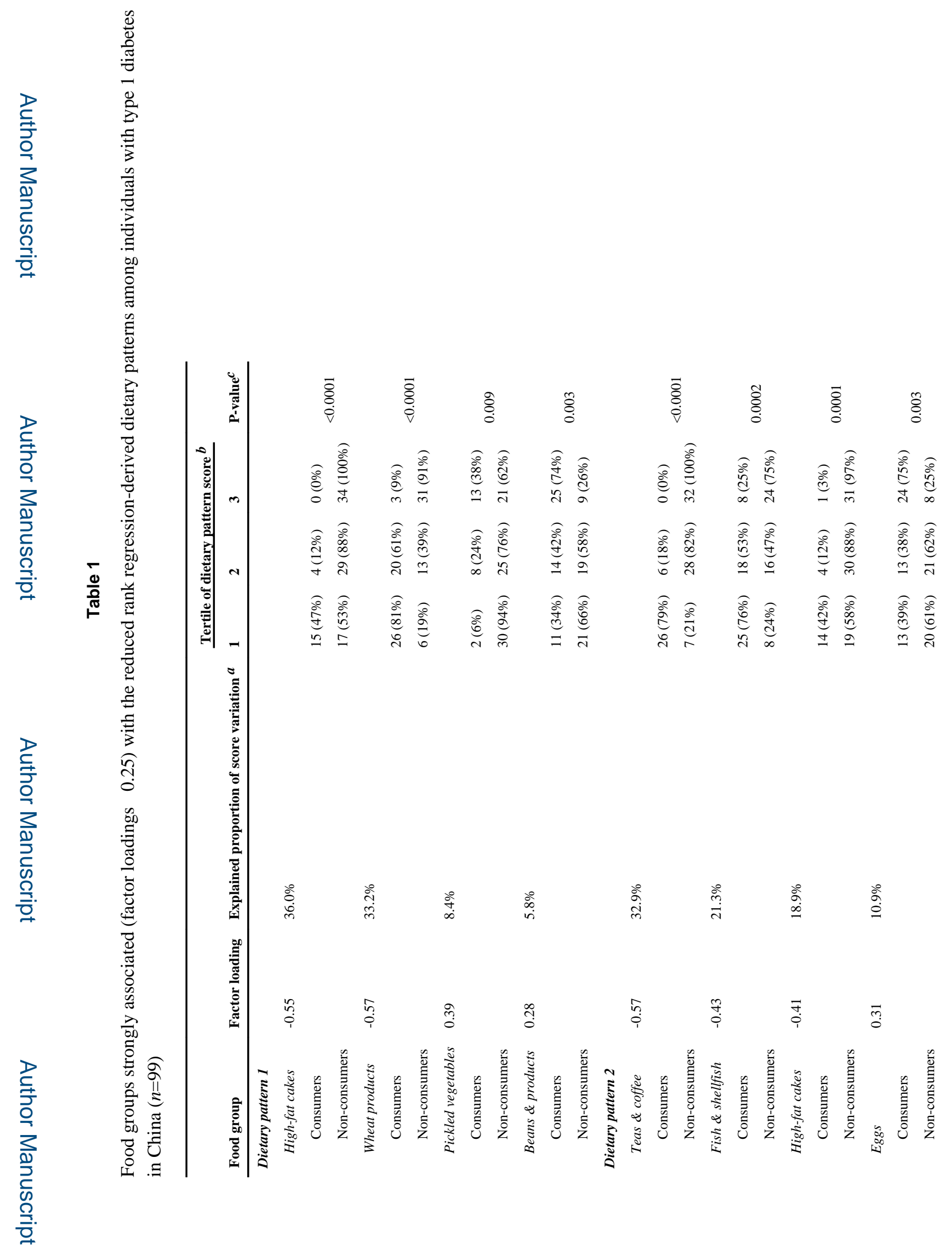

J Diabetes Complications. Author manuscript; available in PMC 2016 April 01. 


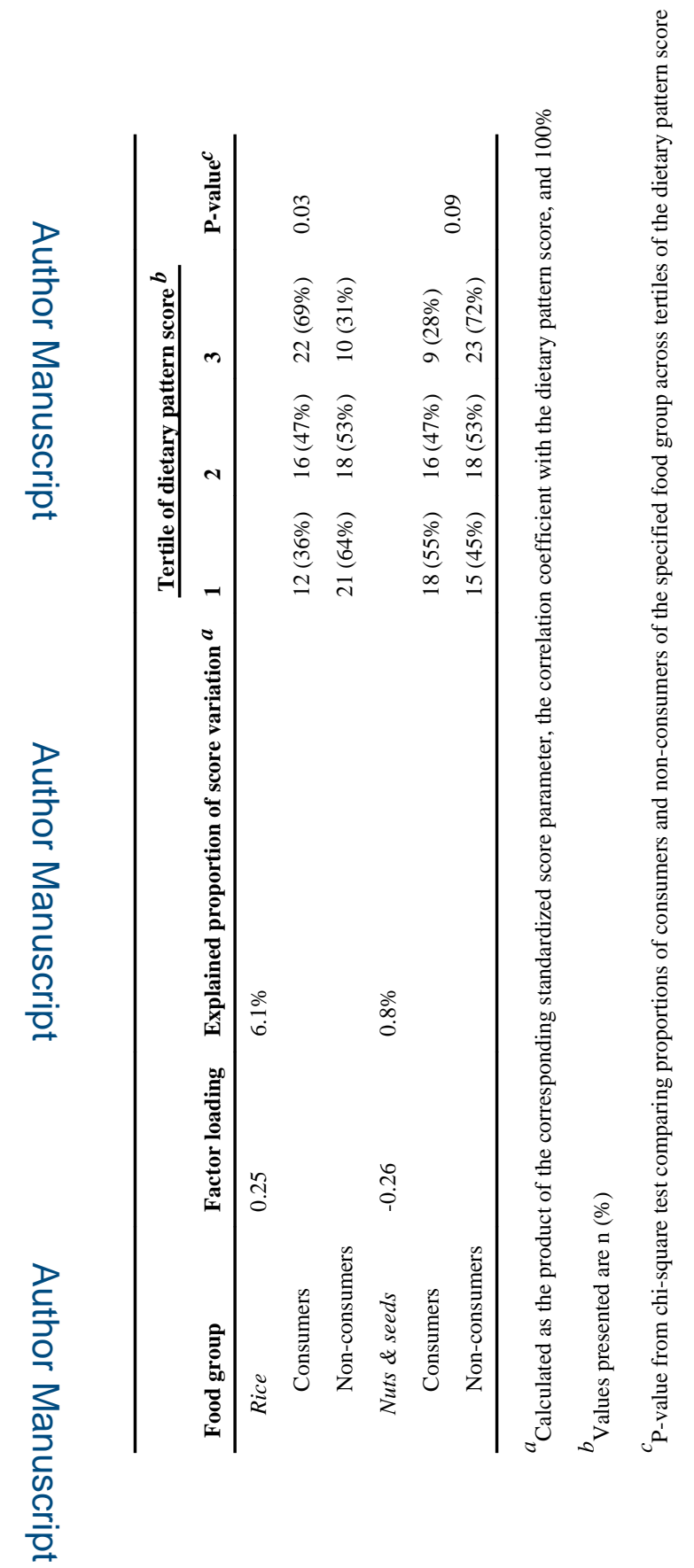




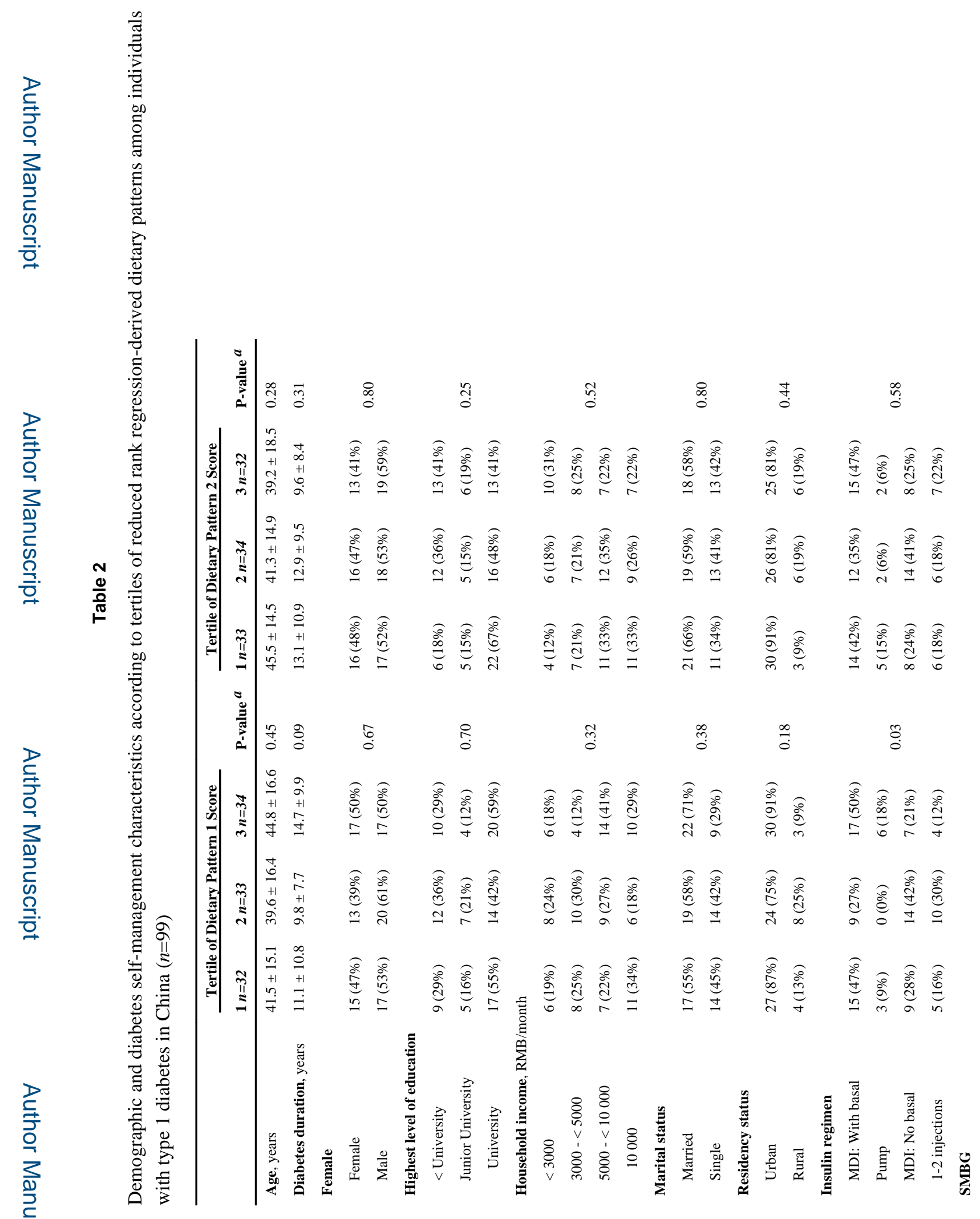




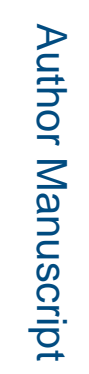

栾

$$
\text { }
$$

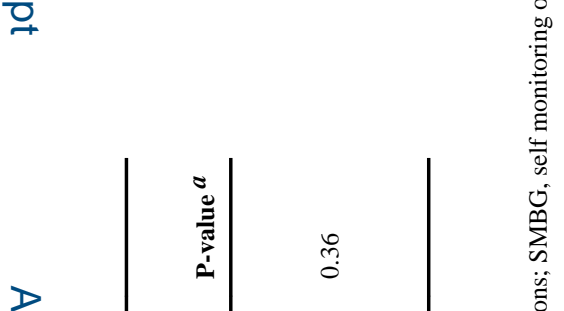

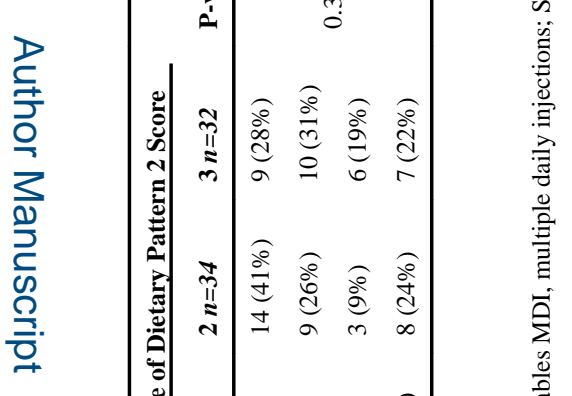



J Diabetes Complications. Author manuscript; available in PMC 2016 April 01. 
Table 3

Bivariate associations of food groups that loaded heavily (factor loadings $\searrow 0.25$ ) on the reduced rank regression-derived dietary patterns with HbA1c and LDL cholesterol among individuals with type 1 diabetes in China $(n=99)$

\begin{tabular}{|c|c|c|c|c|}
\hline & $\mathrm{n}(\%)$ & $\operatorname{HbA1c}^{b}(\%)$ & $\mathrm{HbAlc}^{b}(\mathrm{mmol} / \mathrm{mol})$ & LDL cholesterol $b(\mathrm{mmol} / \mathrm{L})$ \\
\hline \multicolumn{5}{|l|}{ Food groups } \\
\hline \multicolumn{5}{|l|}{ Beans \& products } \\
\hline Above median & $50(51 \%)$ & $8.1(7.4,9.1)$ & $65(57,76)$ & $2.3(2.0,2.8)$ \\
\hline Below median & $49(49 \%)$ & $7.8(6.6,8.7)$ & $62(49,72)$ & $2.3(1.9,2.7)$ \\
\hline P-value $a$ & & 0.14 & 0.14 & 0.67 \\
\hline \multicolumn{5}{|l|}{ Pickled vegetables } \\
\hline Consumers & $23(23 \%)$ & $8.3(6.7,9.7)$ & $67(50,83)$ & $2.1(1.9,3.3)$ \\
\hline Non-consumers & $76(77 \%)$ & $7.8(7.1,8.8)$ & $62(54,73)$ & $2.3(2.0,2.7)$ \\
\hline P-value $a$ & & 0.44 & 0.44 & 0.55 \\
\hline \multicolumn{5}{|l|}{ Rice } \\
\hline Above median & $50(51 \%)$ & $8.2(7.1,9.2)$ & $66(54,77)$ & $2.3(1.9,2.8)$ \\
\hline Below median & $49(49 \%)$ & $7.7(6.8,8.7)$ & $61(51,72)$ & $2.4(2.0,2.8)$ \\
\hline P-value ${ }^{a}$ & & 0.36 & 0.36 & 0.55 \\
\hline \multicolumn{5}{|l|}{ Wheat products } \\
\hline Above median & $49(49 \%)$ & $7.7(7.0,9.0)$ & $61(53,75)$ & $2.1(1.8,2.7)$ \\
\hline Below median & $50(51 \%)$ & $8.1(7.2,9.1)$ & $64(55,76)$ & $2.5(2.1,2.8)$ \\
\hline P-value ${ }^{a}$ & & 0.37 & 0.37 & 0.03 \\
\hline \multicolumn{5}{|l|}{ High-fat cakes } \\
\hline Consumers & $19(19 \%)$ & $7.3(6.4,8.4)$ & $56(46,68)$ & $2.2(1.9,2.5)$ \\
\hline Non-consumers & $80(81 \%)$ & $8.1(7.3,9.2)$ & $65(56,77)$ & $2.3(2.0,2.8)$ \\
\hline P-value ${ }^{a}$ & & 0.008 & 0.008 & 0.41 \\
\hline \multicolumn{5}{|l|}{ Nuts \& seeds } \\
\hline Consumers & $43(43 \%)$ & $7.7(7.0,8.7)$ & $61(53,72)$ & $2.3(2.0,2.8)$ \\
\hline Non-consumers & $56(57 \%)$ & $8.2(6.9,9.4)$ & $66(52,79)$ & $2.2(1.9,2.8)$ \\
\hline P-value ${ }^{a}$ & & 0.30 & 0.30 & 0.60 \\
\hline \multicolumn{5}{|l|}{ Eggs } \\
\hline Above median & $50(51 \%)$ & $8.2(6.9,9.6)$ & $66(52,81)$ & $2.3(1.9,2.6)$ \\
\hline Below median & $49(49 \%)$ & $7.8(7.0,8.8)$ & $62(53,73)$ & $2.4(2.0,2.8)$ \\
\hline P-value ${ }^{a}$ & & 0.33 & 0.33 & 0.48 \\
\hline \multicolumn{5}{|l|}{ Fish \& shellfish } \\
\hline Consumers & $51(52 \%)$ & $7.8(6.7,8.9)$ & $62(50,74)$ & $2.4(2.0,2.8)$ \\
\hline Non-consumers & $48(48 \%)$ & $8.0(7.5,9.1)$ & $64(58,76)$ & $2.1(1.8,2.8)$ \\
\hline P-value ${ }^{a}$ & & 0.37 & 0.37 & 0.08 \\
\hline \multicolumn{5}{|l|}{ Teas \& coffee } \\
\hline Consumers & $32(32 \%)$ & $7.7(6.8,9.0)$ & $61(50,74)$ & $2.5(2.1,3.2)$ \\
\hline Non-consumers & $67(68 \%)$ & $8.2(7.1,9.2)$ & $66(54,77)$ & $2.3(1.9,2.7)$ \\
\hline
\end{tabular}




\begin{tabular}{|c|c|c|c|c|}
\hline & n $(\%)$ & $\operatorname{HbA1c}^{b}(\%)$ & $\mathrm{HbAlc}^{b}(\mathrm{mmol} / \mathrm{mol})$ & $\mathrm{LDL}$ cholesterol ${ }^{b}(\mathrm{mmol} / \mathrm{L})$ \\
\hline P-value $a$ & & 0.23 & 0.23 & 0.05 \\
\hline
\end{tabular}

${ }^{a} \mathrm{P}$-value from Kruskal-Wallis test

${ }^{b}$ Values are median $\left(25^{\text {th }}\right.$ percentile, $75^{\text {th }}$ percentile) 